\title{
Hydromechanics, Aerodynamics and Thermodynamics: Critical Numerical Analysis of Aerodynamics of BLE Turbine Blade
}

\author{
Shih-Chau Lin \\ Department of Mechanical and Mechatronics Engineering, National Ocean University, Taiwan. \\ echaulinshih@outlookmail.com
}

\begin{abstract}
Article Info
Journal of Machine and Computing (http://anapub.co.ke/journals/jmc/jmc.html)

Doi : https://doi.org/10.53759/7669/jmc202101003

Received 14 October 2020; Revised form 22 November 2020; Accepted 25 December 2020.

Available online 05 January 2021.

(C)2021 The Authors. Published by AnaPub Publications.

This is an open access article under the CC BY-NC-ND license. (http://creativecommons.org/licenses/by-nc-nd/4.0/)

Abstract - This contribution presents a numerical analysis based on the effects of aerodynamics of the bump-based humpback whale fins available on the turbine blade edge. In this research, performance comparisons have been made based on dual sequestered blades. One of the blades was sinusoidal in shaped with Bumped Lead Edge (BLE) and the other one with Upright Leading Edge (ULE). However, all the blades are based on a similar cross-sectional profile i.e. NACA-012. This research has been based on simulations of Reynold's number i.e. 1.8.105 of Attack Angle (AA) i.e. from ' $0{ }^{\circ}-30^{\circ}$ '. At this angle, especially greater than $10^{\circ}$, the BLE has indicated an enhancement in about 3.5\% to $9.0 \%$ lift and a reducing drag whereas the negligible variation in lifts and minor drag is displayed for AA less than $10^{\circ}$. The findings in this result for BLE have indicated a substantial achievement in aerodynamic features for particular AA.
\end{abstract}

Keyword - Bumped Leading Edge (BLE), Upright Leading Edge (ULE), Attack Angle (AA)

\section{INTRODUCTION}

Significant exhaustion of fossil fuels has led to negative environmental effects, which has amounted to the fuels becoming expensive as time elapses. Resultantly, this has led to the necessity to invent substitute energy sources. Enhancing conversion efficiency of renewable sources of energy into applicable form i.e. electricity as a priority. Siteparticular optimization of turbines could enhance the application of wind energy and it necessitates the blade design that has been optimized. A numerical analysis into the effect of aerodynamic of radical transformation in turbine blade geometry is considered in this research. The purpose is to evaluate the possibility of enhancements initiated to traditional blades design by incorporating a collection of sinusoidal bumps into Bumped Lead Edge (BLE) of conventionally Upright Leading Edge (ULE).

Numerous technological breakthroughs come from applications of innovative assumptions hence scholars discovered other possibilities of mimicking the condition of BLE stems from tubercles [1]. These are the features protruded on humpback whale's pectoral fin. Megaptera novaeanglia are dangerous mammal species. Baleen whale is agile due to their large size that is about 39 tons and 16 meters in length. During the process of feeding, they can swim swiftly in spiral patter and in tight diameters that are less than 10 meters. Their agility is based on their pectoral fins that are articulated with features featuristic tubercles on BLE, which permit them significant manoeuvrability whenever pursuing plankton, minor shoal of fishes and krill.

The incredible hydrodynamic merit can be considered functional adaptation necessitated through thousands of years based on natural selection of humpback fish and their transformation into significantly skilled hunters as now. The fin bumps have been the major focus of research for potential application in enhanced design for vortex generator. Generally, bumped blade gave a significant advantage in lifts to the drag ration i.e. about $50 \%$ over the Attack Angle (AA) that range from $0^{\circ}$ to $20^{\circ}$. Sinusoidal lead edge delayed stall challenges, enhancing crucial AA by $5^{\circ}$ over the blade one with Upright Leading Edge (ULE).

The influence of dissimilar surface rigidity on aerodynamics of turbine blades is has widely been evaluated in the past literature [2]. Surface rigidity of airfoils has a fundamental implication on the overall loss of energy because skin or fin friction and normally amounts to an enhanced thermal load. There are numerous assumptions have to be done experimentally. For blades to be designed that accommodates contradicting necessities of aerodynamics and manufacturing 
segments of surface rigidity. A completely automatic design procedure based on genetic algorithms has been formulated and results shown in past literature sources. The design blade effectively attained a particular requirement.

Other researchers have evaluated numerical researches using low Reynolds methodology is done to evaluate this effect of non-uniform rigidity applicable to various positions on suction sides of a stress turbine blade. It is indicated that rigidity applicable at trailing and leading edge does not fundamentally affect the flow while rigidity at $25 \%$ cord length and at midchord induce change. Particularly, surface rigidity at $25 \%$ chord length indicates a firm association to the transitions of general loss of pressure.

S. Derakhshan and A. Tavaziani in [3] have assessed the performance of aerodynamic of turbine blade based on overall stress loss whereby measurements were based on linear cascades. Reynold numbers varied from $600 \mathrm{~K}$ to $1.2 \mathrm{M}$ to obtain the operating regimes for heavier duty turbines. In this analysis, Four various forms of surface rigidity on similar profiles were evaluated in high-speed cascade.

The overall stress/pressure loss is considered based on wake traverse calculations. The loss advances because of the surface rigidity to enhance Reynolds numbers. In the analysis, the ultimate Reynolds number i.e. $1.2 \mathrm{M}$ considered an increase in the overall stress loss to ultimately evaluated surface roughness figure of $\mathrm{Ra}=12 \mu \mathrm{m}$ was considered to be $39.9 \%$ contrasted against hydraulically smoothened surfaces. The findings from the calculations were contrasted against the relevant sources of literature. An agreement was reached for higher Reynolds number between the test findings and correlation of relevant literatures.

Earlier on, tests were done at the Illinois University regarding the low turbulence sub-sonic wing tunnel as shown in Fig 1 below. The tunnel of wing is an open and return form contracting ratio of 7:5:2. The rectangular test part is $0.80 .20 \mathrm{~m}$ (approximately $3 \mathrm{ft}$ ) in its cross section with length of about 8.0 feet. Along these lengths of tests, the width enhances by about $1.3 \mathrm{~cm}$ to consider the layered boundary and development in tunnel wall. The test part speed is $71.5 \mathrm{~m} / \mathrm{s}$ through 93.3 $\mathrm{kW}$ that alternates the current electric motors that drive a fan with five blades. The setting of the tunnel chamber is about $10 \mathrm{~cm}$ (thickness of honeycomb) and in this case, a turbulence screen can be considered. Ultimate Reynold numbers were $4.9 \mathrm{million} / \mathrm{m}$

Dynamic stress and airspeed in the test segment were assessed based on static evaluation of pressure in the tunnel. Ambient temperature was assessed using thermocouple. Axial pressure, pitched airfoil moment and normal force was measured based on the application of three element external force and moment balancing that has been mounted beneath the test segment. The design was then mounted based on the span-wise axis in a vertical locus.

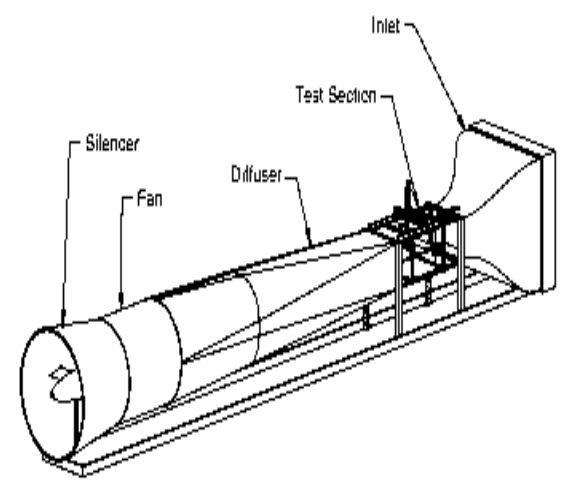

Fig 1: Low turbulence sub-sonic wing tunnel

Calculations of drag and lift were done from axial and normal forces, but accurate drag figure was considered from the wake rake evaluations. Rake incorporated 58 overall stress probes against the general width of $24.8 \mathrm{~cm}$. Seven probes on every outer sides of rakes had been spaced $6.9 \mathrm{~mm}$ apart and the remainder interior probe was placed $3.40 \mathrm{~mm}$ apart. About eight span-wise wake profiles were evaluated and every AA from $10.10 \mathrm{~cm}$ and ending with $7.60 \mathrm{~cm}$ beneath the center span and the resultant local drag value were based on the mean average. The wake-rake drag measurement was then reported in their analysis. The calculations were then rectified for the wind tunnels' effect before being validated through the comparison information considered for S-809 model of airfoil with information captured at the Ohio State and Delft University.

In this paper, a numerical evaluation of the aerodynamic features of BLE turbine has been done. This Section has introduced an analysis into the effect of aerodynamic of radical transformation in turbine blade geometry is done. The purpose is to evaluate the possibility of enhancements initiated to traditional blades design by incorporating a collection of sinusoidal bumps into Bumped Lead Edge (BLE) of conventionally Upright Leading Edge (ULE). Section II presents a background analysis of the research. Section III focusses on the literature reviews. Section IV presents a critical analysis of the numerical model, which denotes the aerodynamics characteristic of BLE turbine blade. Section V presents the findings while Section VI concludes the analysis and proposes future directions. 


\section{BACKGROUND ANALYSIS}

Because efficacy of turbomachines is centered on the blade's performance in operation dimension, optimum designing of the blade is fundamental. The connection between the performance of hydrodynamic of pectoral fins of humpback whales and the turbine blade aerodynamics can be utilized to structure a blade mimicking the flow influence of fin tubercles. Consequently, fluid flow analyses of secluded blades for wide-range AA are presented. Two forms of blades have been evaluated: the first one represents the linear aerodynamic extrusion of airfoil profile with smoothened BLE whereas the second one is based on the same cross-section but with sinusoidally curved BLE based on tubercles.

Assumptions were that curvature would influence turbulent separations of airflows from blades that occur at high AA and in this manner; a significant lift force is produced. Due to irregular geometry and BLE blade curvature and dominant 3D fluid flow influence close to the wake areas, it was fundamental to undertake $3 \mathrm{D}$ evaluation of the flow. This methodology gave accurate contrast of dual blades. If BLE blade has to indicate effective performance features compared to the straight one, this form of modification could identify the use in significant dimensional applications such as water turbines, wind turbines, fans, helicopter rotors, airplane wings.

\section{LITERATURE REVIEW}

G. Dhiman and A. Kaur in [4] evaluated the different airfoil designs for wide-range Reynolds number and AA. The tests for wind tunnels are the factual approach for validating the numerical evaluations airfoil performances. H. Kawazoe and S. Kato in [5] simulate the flows of the wing based on BLE and the other one without them. However, all of them were based on significant Reynolds number and varied AA. They were noted with reference to the enhancement in wing performances with BLE tubercles. For AA at approximately $10^{\circ}$, the development in the resultant lift was about $5 \%$ whereas the drag reduction was $11 \%$. The ratio of lift drag enhance by about $18 \%$.

Y. Yuta, O. Tomohisa and M. Akinori in [6] evaluated the NACA-012 airfoil with the length of chord, $\mathrm{c}=0.12$, based on the varied sinusoidally designed tubercles with sine amplitude that ranges from $0.031 \mathrm{c}$ to $0.751 \mathrm{c}$ and sine wavelengths by $0.21 \mathrm{c}$. The findings of their evaluations based of their evaluated, performed based on Reynolds number 1.23 .105 indicated that the diminishing in the wavelength of tubercles amounted to fundamental AA enhancement and that the diminishing tubercle amplitude enhanced the lift. Wings attained maximum critical AA of $15^{\circ}$ for minor wavelengths (of about $0.02 \mathrm{c}$ ) and the ultimate lift for minimal amplitude that is fundamentally smoothened LE wing.

H. Yamanishi, Y. Tanaka and S. Murata in [7] presented experimental contrasts between unmodified NACA-012 airfoil and the same one with the tubercles of different amplitudes i.e. $0.03 \mathrm{c}$ to $0.12 \mathrm{c}$ and wavelength of $0.44 \mathrm{c}$, with reference to the length of the chord of approximately $70 \mathrm{~mm}$. Reynolds number was 1.2.104. The results of their evaluation are noted that minor Reynolds in tubercles foils have delayed stalls where the enhancements in performance were minimal and insignificant.

V. Gopinathan, J. Bruce Ralphin Rose and M. Surya [8] argued concerning tubercle efficacy based on Reynolds number. They evaluated the influence of sinusoidal structured BLE of NACA-012 airfoil to create comparisons on the findings with ULE. The number of Reynolds was 5.7.105, the length of the chord was $\mathrm{c}=0.1 \mathrm{~m}$, the amplitude was $0.25 \mathrm{c}$ and 0.05 whereas wavelengths were considered at $0.50 \mathrm{c}$ and $0.25 \mathrm{c}$. The researchers structured these simulation and structured the experimental validations to make the results significantly matched.

J. Yeston in [9] argues that wings with shorter wavelengths and the smaller amplitudes is denoted by consistent features for AA less than $13^{\circ}$ whereby separation starts and wings stall. For AA more than $15^{\circ}$, lists on tubercles was more than $47 \%$ more and drags more than $43 \%$, which is less compared to the ordinary wing. The findings identified that an amplitude of wing bumps fundamentally based on their performance for higher AA since the ultimate lift was attained at optimum AA since maximum lift was attained at optimum AA through ULE and conventional wing.

A. Sareen, C. Sapre and M. Selig in [10] have evaluated the findings in their study of the effects of edge erosions on the performance of aerodynamics of airfoil turbines. Evaluations are done on the turbine airfoils at Reynolds of $1 \mathrm{M}$ and AA spanning at nominal lower drags dimensions of airfoils. The airfoils have been assessed based on simulation. Eroded Leading Edge (ELE) based on the varied erosional severity to evaluate the loss in performances because of the ELE.

E. Hastings and G. Manuel in [11] argue that tests also span based on the simulated bug on airfoils to evaluate the implication of insects' accretion based on the performances of airfoil. The major purpose was to formulate the baseline comprehension of aerodynamics influence based on varied level of ELE and quantifying their relative implication on the performance of airfoil. Their results indicate that ELE can general substantial performance on airfoil and degradation, hence yielding massive increment in the drag interlinked with critical loss in lifts nearer to the top drag polar that is fundamental for the maximization of the wind energy turbines.

A. Farokhipour, z. Mansoori, M. Saffar-Avval and G. Ahmadi in [12] argue that the first phase in modeling erosion was to comprehend the manner in which erosion develops on turbine blades. This is the phase where images of eroded blade produced by $3 \mathrm{M}$ Reynolds are applicable. Image data collected by $3 \mathrm{M}$ from energy plant operations considered a significant dimension of motor blade sizes (incorporating mega-scale rotor), which has been operated for more than a decade. These photographs exposed that erosional process begins with the creation of small holes close to the lead edge of the blades. As the pit density advances with time, they link up to create deeper and larger gouges. 
D. Serson and J. Meneghini in [13] argue that delamination that begins at the leading edges and develops in the chordwise extent as time goes, which makes it the last phase of erosion. Two fundamental observations are considered in the research. The first one is that The densities of gouges and pitches is close to the leading edge and diminishes based on the chord directions. The second incorporates critical impingement and thus amounts to erosion at the lowest surfaces due to the localized mean AA, which is positive for the operation in wind energy turbines.

A. Sareen, C. Sapre and M. Selig in [14] attest that with reference to the erosion process, tunnel model is evaluated and tested based on various forms of erosion characteristics i.e. BLE delamination, gouges, and pits. Tests were done with category $\mathrm{A}$ i.e. pits and category $\mathrm{B}$ i.e. gouges and pits and finally category $\mathrm{C}$ i.e. BLE delamination, gouges and pits. The categories were considered in the simulation of the erosional process. The dimension of each category was categorized under particular phases with each phase about two numbers of gouges and pits and dual the delamination extent. The diameter and depth of the chord-wise extent of leading edge, gouges and pits delamination was referenced from the downscaled calculations from photographs of ELE by 3.0M. The pit depths, BLE delamination and gouges was considered to be $1 \mathrm{~mm}, 3 \mathrm{~mm}$ and $4 \mathrm{~mm}$ respectively. The mean pit diameter and gouge was structured the same way as the overall depth.

G. Silva, M. Donadon and F. Silvestre in [15] confirm that, to structure the varied chord-wise extent and magnitude of erosion elements on the top part different from the low surface, a 1:1:3 ratio was utilized. This ratio was selected with reference to the observation from photographic information. With reference to the ratio, chord-wise extent of gouges and pit was structured for the scenarios at $\mathrm{s} / \mathrm{c}$ at $9 \%$ of the upper segment and $12 \% \mathrm{~s} / \mathrm{c}$ on the lower segment. The gouge number and pits on the lower segment was 1.351 more compared to the upper surfaces. Based on the application of a similar ratio, the dimension of BLE delamination was structured to be $\mathrm{s} / \mathrm{c} 2 \%, 3 \%$ and $4 \%$ on the top surface and $1.5 \%$, $2.7 \%$ and $4.0 \%$ on the low surface.

\section{CRITICAL NUMERICAL ANALYIS}

Wind-energy turbine blades are typically exposed to rain and precipitation, which happen in different forms [16]. Myriad abrasive airborne materials can possibly erode surfaces, i.e. BLE. The materials can develop erosion on the blade and minimize the performance of aerodynamics and therefore, the capturing of energy. In addition, in the same ecosystem, the debris of insects and airborne materials can accrete on BLE of the turbine blade. BLE erosion and accretion on the blade, including contaminations can fundamentally minimize the performance of the blade certainly in high-speed rotor tip part, which is significant in the optimization of the blades' performance and the capture of energy.

The erosion procedure on wide-energy turbine blade normally begins from the formation of minor pits next to BLE, which enhances the time density and links that create gouges [17]. When dedicated to nature, gouges can potentially develop in size, density and link up to cause delamination next to BLE. Fig 2 displays the damage extent that amounts to the erosion of BLE on the turbine blades in operations. Fig 2 (a) shows the gouges blades and pits close to BLE while Fig 2 (b) indicates an older blades with delamination surpassing the full leading edge.

Even if detrimental BLE erosion condition is known in the engineering industry, some efforts have been considered in the quantification of the erosion effects on the performance of turbines. M. Virk in [18] has concentrated on the acceleration of dust, insect debris, and ice and DLE rigidity generally on wing turbine blade and not on blades' erosion. In addition, wide-range researches have based on qualitative evaluation of data on accretion of turbine performance. The researches about erosions have widely utilized rigidity strips or zigzag tapes applicable close to BLE of airfoil for surface simulation and contamination. However, the methodology is widely applied since it is simply and does not necessitate high degree of accuracy hence forming erosion on the wind turbine blades. The modification of shapes is typically negative, which means it erodes off.

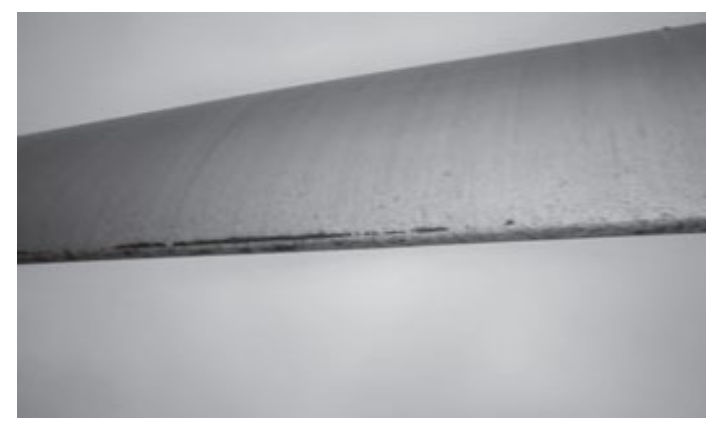

a) 


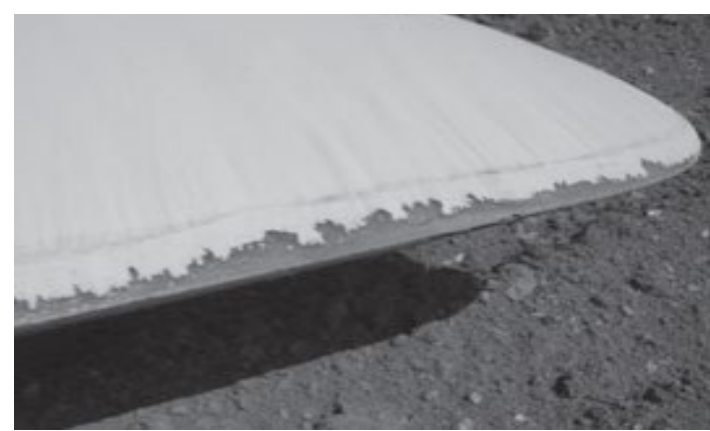

b)

Fig 2: (a) Blade with gouges and pits close to BLE; (b) Old blade with delamination over the complete leading edge

A. Gross, H. Fasel, T. Friederich and M. Kloker in [19] focused on an analysis that tests the turbine airfoil based on the modification of shapes to simulate BLE erosion based on evolution stages of the developments. Surface erosion has been modelled based on the observation of photographic record. The obligation of developing a baseline comprehension of the effects of aerodynamics of different forms and degree of BLE erosion is to count relative implication of the performance of airfoils. The purpose of their research was to evaluate the turbine airfoils with shape change for the simulation of BLE erosion via different evolution phases of development. The erosion against the surface has been modeled based on the observations of photographic records. The main purpose was to conduct an analysis that investigate the detrimental influence of BLE erosion and the necessity for continued development of erosion mitigating approaches.

\section{A. Numerical Model: Mesh and Blade Geometry}

This analysis focuses on the implication of BLE on aerodynamic features in isolated flow fields. The impact of bumps was evaluated based on the comparison of particular numerical lift-drag features between BLE and the ULE. The crosssection of the blades were referenced to NACA-012 airfoils profile. Fig 3 below displays the geometrical definition of BLE
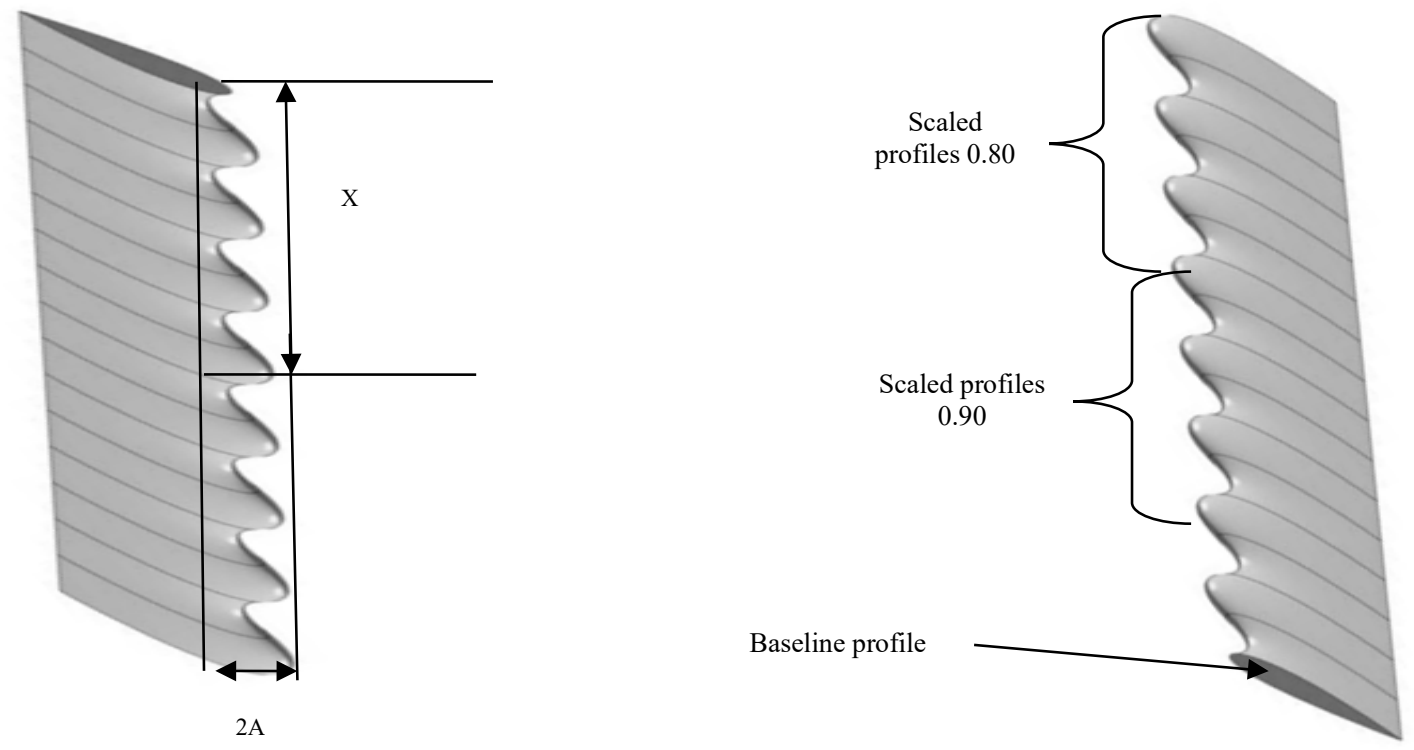

Fig 3: Geometrical definition of BLE (indicating amplitude and wavelength)

BLE was linked based on three-scaled NACA-012 profile and mutually spaced in a vertical manner in the sinusoidal wave pattern hence making tubercle-based bumps in BLE. The chord of the profile was structured at $100.75 \mathrm{~mm}$ for the larger one, second one and third one scaled at 0.90 and 0.80 chords' length. The resultant multi-sectional surfaces formed from the three profiles incorporated eight $39 \mathrm{~mm}$ wide segments of periodic blades, which had been attached to create BLE model as indicated in Fig 4 below that also indicated the comparison of BLE of dual blades: ULE and BLE. The varied dimensions of ULE were minimized to $92 \%$ of the BLE so that similar surfaces of the blades could potentially be obtained in both instances. 


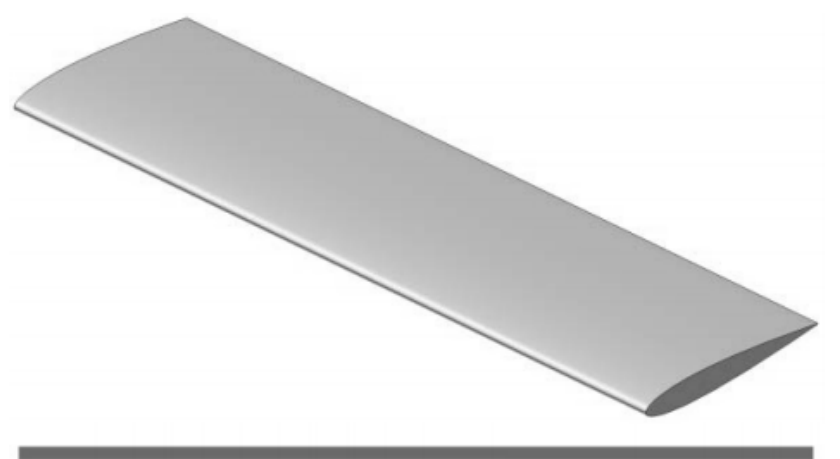

a)

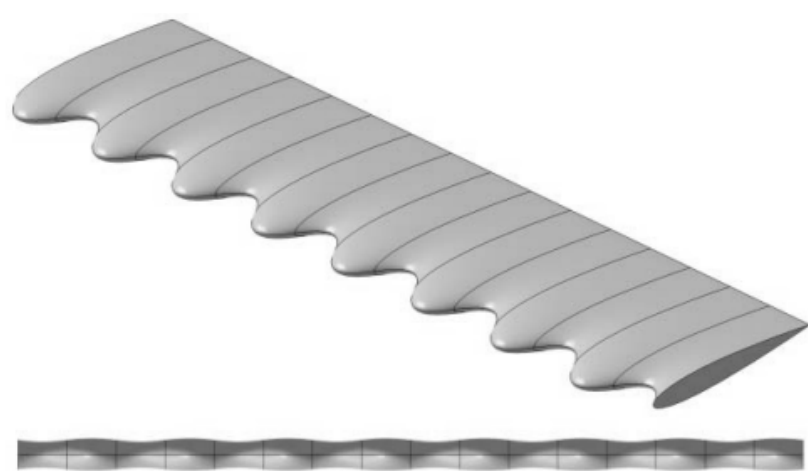

b)

Fig 4: Comparison of BLE of dual blades: (a) ULE and (b) BLE

The last blade surface segment evaluated in modeler was 0.03 square meter. Table 1 includes the geometrical features of the evaluated blades.

Table 1: Geometrical features of ULE and BLE

\begin{tabular}{lcl}
\hline & ULE & BLE \\
\hline Profile blade length & 0.94 Lchord & Lchord $=100 \mathrm{~mm}$ \\
Amplitude & - & 0.1 Lchord \\
Wavelength & - & $0.4 \mathrm{Lchord}$ \\
Repeat part, vertical depth & $319 \mathrm{~mm}$ & $39 \mathrm{~mm}$ \\
Blade overall vertical depth & $319 \mathrm{~mm}$ & $319 \mathrm{~mm}$ \\
Blade cross-sectional areas & $0.03 \mathrm{~m} 2$ & $0.03 \mathrm{~m} 2$ \\
\hline
\end{tabular}

The geometry of the blades has been modelled using catia and the blades have been scaled to the required dimensions that have been positioned so that their trail edges are found at the coordinate model. This is when imported to the Gambit processor or mesher. Fig 5 indicates the computational domain utilized for fluid flow simulation. Fig 5 (a) and (b) have been presented based on the rigidity of the tested numerical mesh with the purpose of providing clear views of resultant hexahedral volume. 


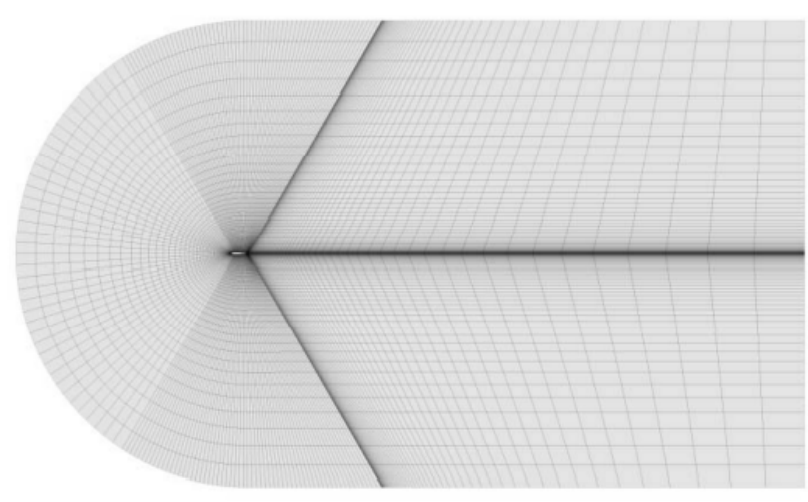

a)

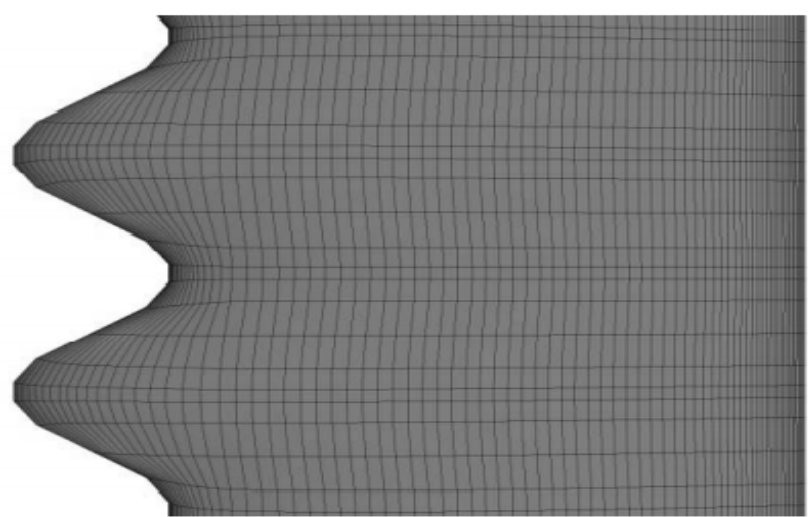

Fig 5: Numerical mesh represented on (a) top wall (b) surface of the blade

The inlet part is found about 13 Lchord upwards from BLE of the blade whereas the outlet part is found 29 Lchord away from the trail edges of the blade to minimize the reflection of the disturbances from the boundaries of the outlets. The lower and upper computational domain boundary is found 13 Lchord further from trail edge. Computational domain was formed based on the application of Gambit processor.

Numerical mesh was produced using C-type discretization system whereas computational domain is defined based on reasonable number of hexahedral component, which is the structured mesh. The findings in the minor size of the numerical mesh were for a particular dimension of solution accuracy. The computational domain was grouped into six different domains to control the overall system application projected for high-quality numerical mesh with hexahedral component since this will ensure fast and safe solution convergence.

C-type system provides simplified discretization controls of numerical mesh refinements ie the control cell number and the stretch element stimulate the fine sized adjustment for the first cell closer to the wall that gives the $\mathrm{Y}+$ value within the required dimension necessitated by the turbulence model.

\section{B. Computational Model}

Commercial liquid flow solver utilized to undertake numerical evaluation where controlling calculations have been discretized use standardized finite volume approach. The mathematical model is made to be simple because it is considered that the flow of fluids is without the transfer of heat. Turbulence is considered since the evaluated flow of fluids fall into the highest Reynolds number where intensive changes of turbulent physical quantities happen. The modest approach of incorporating turbulence is Reynolds mean as the controlling mathematical model.

\section{1) Conservation Calculation}

After evaluating turbulence using the Reynolds mean method, incompressible flow is considered based on Navier stokes calculation as shown in the equations below:

$$
\begin{array}{r}
\frac{\partial v_{i}}{\partial x_{j}}=0 \\
\rho \frac{\partial v_{i}}{\partial t}+\rho v_{j} \frac{\partial v_{i}}{\partial x_{j}}=
\end{array}
$$



as:

The pressure of Reynolds is linked to the average speed gradient based on Boussinesq hypothesis, which is expressed

$$
\begin{gathered}
=-\frac{\partial p}{\partial x_{i}}+u \frac{\partial v_{i}}{\partial x_{j}}+\frac{\partial p}{\partial x_{i}}\left[\rho v_{i} v_{j}\right] \\
{\left[\rho v_{i} v_{j}\right]=u \frac{\partial v_{i}}{\partial x_{j}}+u \frac{\partial v_{j}}{\partial x_{i}}-\frac{2}{3} \rho k \delta_{i j}}
\end{gathered}
$$

The k- $\varepsilon$ standard turbulence scheme with developed wall treatment is chosen for the closure of turbulence. The remedydependent parameter with no dimension $(\mathrm{Y}+)$ was utilized as the recommended measure for numerical mesh quality whereby every simulation is maintained within the projected dimension on the wall blade zones.

\section{Conditions of Boundaries}

The type of mesh considered in Fig 5 indicates the blade position against the domain edge. In this case, the conditions chosen for the domain boundary during the numerical evaluations of the fluid flow of the blades include the speed inlet conditions, which are chosen for the round inlet segment and the elements of velocity for every AA that was analyzed. The speed magnitude was about $\mathrm{vm}=25 \mathrm{~m} / \mathrm{s}$. The turbulent dissipation and kinetic energy was based on the intensity of turbulence, which is about $3 \%$ and the diameter of hydraulic (which determines the length of the blades).

The outflow boundaries' conditions have been chosen at the rectangular outlet part. The surface of the blade has been illustrated as non-ship and non-porous boundary where fluid speed reaches zero and its roughness considered as well. The remaining boundary of the domain delimits the air volume around the blades and is defined as non-periodic wall of the slip to minimize the effects on computational results. The fluid volume illustration utilized the standardized feature of gaseous air that was chosen from the solvers' database. In this case, gravity was considered and the operating stress was considered as per the normal atmospheric pressure.

\section{Convergence}

The values and residual for coefficients of lift-drag have been monitored during the entire numerical evaluation. The convergence method was satisfied when the values of critically evaluated residuals decreased below 10 , which represents two magnitude orders less than the standardized convergence methods, and the computational values of lift-drag coefficient that has been stabilized against iterations. Normal transformations in residuals and coefficients of the lifts during the process of iterations are displayed in Fig 6. It is considered that the convergences for this was attained after completing more than 500 iterations.

\section{FINDINGS}

Lift-drag stress are two fundamental variables to show the quality of aerodynamics of a particular design of blade. The efficiency of the blades is enhanced based on the increment of the lifts and minimized drags. The designers typically grade the profile quality and shape based on the dimensionless lift co-efficient (LC) and drag co-efficient (DC).

$$
\begin{gathered}
C_{l}=\frac{l}{1 / 2 \rho \infty v \frac{2}{\infty} A} \\
C_{d}=\frac{d}{1 / 2 \rho \infty v \frac{2}{\infty} A}
\end{gathered}
$$

Whereby L represents the pressure of the lift, D represents the drag pressure; A represents the blade area whereas v $\infty$ is the free streaming velocity. To evaluate the aerodynamics quality of the assessed blade, Fig 6 (a) and (b) indicate a basic comparison of the transformations in drag-lift co-efficient against AA for BLE and ULE blade. The selection between the dual forms of blades is dependent on acceptable lift-drag ratio against the complete range on the mean or the optimal segment of AA.

Based on information represented in Fig 7, it can be summarized that, for an enhanced AA from the $0^{\circ}$ to $15^{\circ}$, the coefficient of the lift enhances for the tested blade. The variation in the computational lift co-efficient between the blades is considerably minimized, so it can be considered that the application of bumps on BLE of the profiles will have negative implication on this segment. Since the AA range is specified as close or optimal to the optimum working segment of turbomachine, it is fundamental to consider that blade bumps BLE have no fundamental adverse effect on the lift coefficient in this segment.

Enhancing AA more than $15^{\circ}$, until the maximum examined aspect i.e. $30^{\circ}$ leads to the positive implication of BLE. In this AA range, the lifts co-efficient is $3.5 \%$ to $10 \%$ more for BLE compared to ULE (Fig 7). Resultantly, utilizing the model of k- $\varepsilon$ turbulent makes the computation value of lifts for the blade to signify occurrence staff at high AA compared 
to the figures obtained experimentally. Effective results have to be projected using the model of turbulence with high degrees of accuracy based on this model to demonstrate the advantages of BLE.

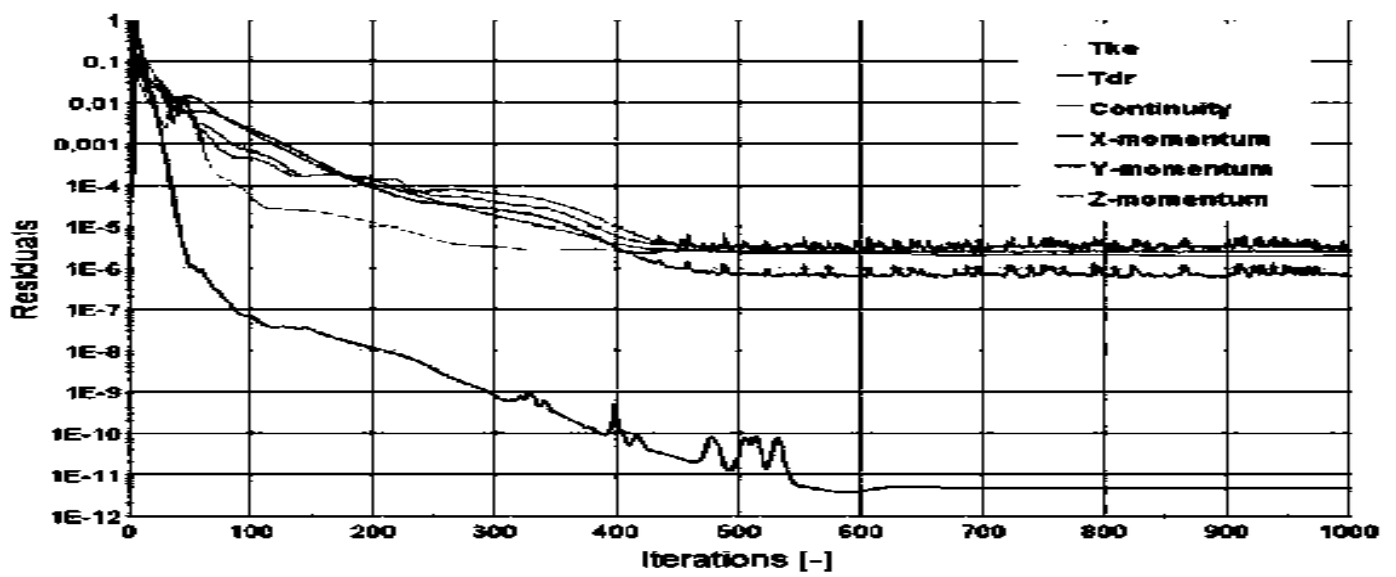

a)

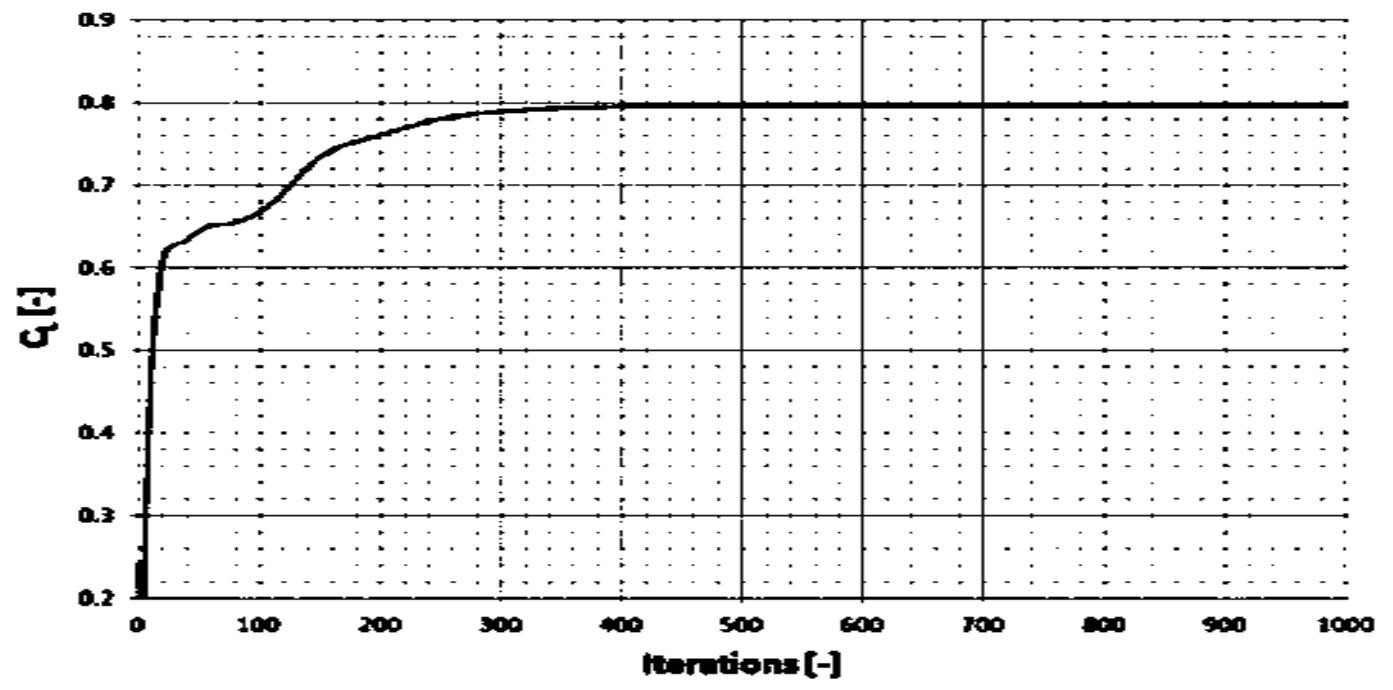

Fig 6: (a) Residuals convergence and (b) monitored lift values of co-efficient during iterations

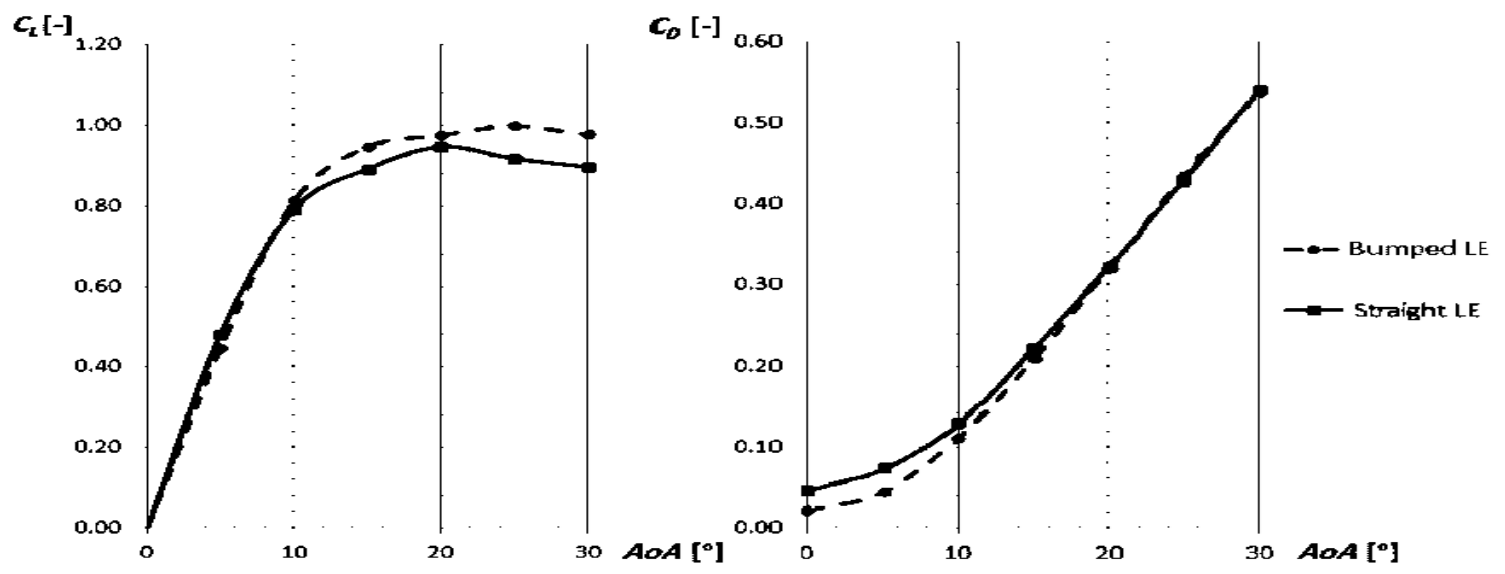

Fig 7: Co-efficients (a) Lift LC (b) Drag DC as for the BLE over AA

Comparison of drag co-efficient will indicate that DC is minimized for lower AA i.e. $0^{\circ}$ and that its development is positive and proportional to the enhancement of AA in all the directions (See Fig 7). In the range of AA from $0^{\circ}$ to $15^{\circ}$, the same DC value was evaluated for BLE. This justifies the application of BLE in segments of optimal AA as drag co- 
efficient that was computer was more than $18 \%$ for BLE than ULE. The drag co-efficient for the parts about $\mathrm{AA} 15^{\circ}$, a similarity in the blades was considered and BLE provided no relative advantage in this case. DC simulation providing integral features of the evaluated issue permits computing visualization of the fluid flow. When the flow surpasses the bump, dual longitudinal vertices are created and to direct the flow of fluids to the suction part of the blades due to secondary rotating flow element. The feature of bumped BLE leads to potential delays in the stall issues and effective aerodynamics characteristics.

\section{CONCLUSION AND FUTURE DIRECTIONS}

In conclusion, the assumptions that the shape of BLE of the humpback whale flip formulated by the transformation of cetacean has fundamental influence on aerodynamic properties at high AA are justifiable. Significant AA of fines of the whales happen during the process of feeding when rapid transformation in their direction of movement is critical for effective feeding. To effectively regenerate this, a wide-range AA was evaluated to produce a compared features computed through the simulation of fluid flow on ordinary ULE and whale-based blades with BLE whereby isolation is considered in a three-dimensional flow field.

Evaluations in this paper indicate that the mathematical approaches on co-efficient lift for curved BLE is more for the evaluated AA range i.e. $0^{\circ}$ to $30^{\circ}$ except for narrow parts at low AA when ULE blades were assigned high lift co-efficient as the flow of fluids around the blades has been optimized. Critical lift co-efficient on BLE is noticed in segments more than $\mathrm{AA}$ of $10^{\circ}$, which is about $3.5 \%$ and $10 \%$ more than the ULE. Enhanced lift co-efficient of BLE also transformed the stall issue to a critical angle i.e. $6^{\circ}$ more than the one known for ULE. DC for BLE is not fundamentally lower in the tested parts outside the optimum level.

The greatest reduction in the drag co-efficient happens for minor AA whereby more economically ULE is advantageous in LC. In general, effective features of LC in the segments above AA of $10^{\circ}$ and effective features of drag co-efficient of parts of low AA affected BLE and indicated fundamental merit over the normal ULE. The enhanced aerodynamics characteristics on BLE discussed in this contribution confirm an in-depth analysis of the BLE shape for rotary mechanical appliances.

In the future, it will be necessary to evaluate the effect of wavelengths and amplitudes of bumps on the properties of BLE blade designs and to differentiate the relations effective fluid flow regimes based on Reynolds numbers. The rotor blade with curved BLE shapes has to present efficiency curves in off-cam segments without affective the optimum segments. This has to be verified using experiments based on the built model that can constitute the future directions of research. The flow evaluations of parameters in wind tunnels would possibly provide further experimental findings that would facilitate the comparisons done based on numerical simulations.

\section{References}

[1]. P. Anderson and A. Vesnaver, "The Leading Edge", The Leading Edge, vol. 36, no. 1, pp. 103-103, 2017. Available: 10.1190/tle36010103.1.

[2]. S. Sakipova, "MODELING AERODYNAMICS OF A WIND TURBINE WITH CYLINDRICAL BLADES IN A TURBULENT AIR FLOW", Eurasian Physical Technical Journal, vol. 17, no. 1, pp. 106-112, 2020. Available: 10.31489/2020no1/106-112.

[3]. S. Derakhshan and A. Tavaziani, "Study of Wind Turbine Aerodynamic Performance Using Numerical Methods", Journal of Clean Energy Technologies, vol. 3, no. 2, pp. 83-90, 2015. Available: 10.7763/jocet.2015.v3.174.

[4]. G. Dhiman and A. Kaur, "Optimizing the Design of Airfoil and Optical Buffer Problems Using Spotted Hyena Optimizer", Designs, vol. 2, no. 3, p. 28, 2018. Available: 10.3390/designs2030028.

[5]. H. Kawazoe and S. Kato, "Effects of Leading Edge Separation Vortex of Flexible Structure Delta Wing on Its Aerodynamic Characteristics(Wing and Airfoil)", The Proceedings of the International Conference on Jets, Wakes and Separated Flows (ICJWSF), vol. 2005, no. 0, pp. 583-589, 2005. Available: 10.1299/jsmeicjwsf.2005.583.

[6]. Y. Yuta, O. Tomohisa and M. Akinori, "1201 PRESSURE DISTRIBUTION ON A NACA0012 AIRFOIL AT LOW REYNOLDS NUMBERS", The Proceedings of the International Conference on Jets, Wakes and Separated Flows (ICJWSF), vol. 20134, no. 0, pp. _1201-1_1201-5,2013. Available: 10.1299/jsmeicjwsf.2013.4._1201-1_.

[7]. $\bar{H}$. YAMANISHI, Y. TANAKA and S. MURATA, "PIV Measurement on Separataion Flow around NACA0012 Airfoil in Periodic Flow at Low Reynolds Number", The Proceedings of the Fluids engineering conference, vol. 2016, no. 0, p. 0213, 2016. Available: 10.1299/jsmefed.2016.0213.

[8]. V. Gopinathan, J. Bruce Ralphin Rose and M. Surya, "Investigation on the effect of leading edge tubercles of sweptback wing at low reynolds number", Mechanics \& Industry, vol. 21, no. 6, p. 621, 2020. Available: 10.1051/meca/2020095.

[9]. J. Yeston, "Short wavelengths birth shorter ones", Science, vol. 350, no. 6265, pp. 1217-1219, 2015. Available: 10.1126/science.350.6265.1217-1.

[10]. A. Sareen, C. Sapre and M. Selig, "Effects of leading edge erosion on wind turbine blade performance", Wind Energy, vol. 17, no. 10, pp. 15311542, 2013. Available: $10.1002 /$ we. 1649 .

[11]. E. Hastings and G. Manuel, "Scale-model tests of airfoils in simulated heavy rain", Journal of Aircraft, vol. 22, no. 6, pp. 536-540, 1985. Available: $10.2514 / 3.45161$.

[12]. A. Farokhipour, z. Mansoori, M. Saffar-Avval and G. Ahmadi, "Numerical modeling of sand particle erosion in return bends in gas-particle twophase flow", Scientia Iranica, vol. 0, no. 0, pp. 0-0, 2018. Available: 10.24200/sci.2018.50801.1871.

[13]. D. Serson and J. Meneghini, "Numerical Study of Wings With Wavy Leading and Trailing Edges", Procedia IUTAM, vol. 14, pp. 563-569, 2015. Available: 10.1016/j.piutam.2015.03.069.

[14]. A. Sareen, C. Sapre and M. Selig, "Effects of leading edge erosion on wind turbine blade performance", Wind Energy, vol. 17, no. 10, pp. 15311542, 2013. Available: 10.1002/we.1649.

[15]. G. Silva, M. Donadon and F. Silvestre, "Experimental and numerical investigations on the nonlinear aeroelastic behavior of high aspect-ratio wings for different chord-wise store positions under stall and follower aerodynamic load models", International Journal of Non-Linear Mechanics, vol. 131, p. 103685, 2021. Available: 10.1016/j.ijnonlinmec.2021.103685.

[16]. B. Joyce, J. Farmer and D. Inman, "Electromagnetic energy harvester for monitoring wind turbine blades", Wind Energy, vol. 17, no. 6, pp. 869876, 2013. Available: 10.1002/we.1602. 
[17]. D. Eisenberg, S. Laustsen and J. Stege, "Wind turbine blade coating leading edge rain erosion model: Development and validation", Wind Energy, vol. 21, no. 10, pp. 942-951, 2018. Available: 10.1002/we.2200

[18]. M. Virk, "Effect of Wind Turbine Blade Profile Symmetry on Ice Accretion", Applied Mechanics and Materials, vol. 863, pp. 229-234, 2017. Available: 10.4028/www.scientific.net/amm.863.229.

[19]. A. Gross, H. Fasel, T. Friederich and M. Kloker, "Numerical investigation of rotational augmentation for S822 wind turbine airfoil", Wind Energy, vol. 15, no. 8, pp. 983-1007, 2012. Available: 10.1002/we.540. 